Pacific Journal of Mathematics

RATIONAL WHITEHEAD PRODUCTS AND A SPECTRAL 


\title{
RATIONAL WHITEHEAD PRODUCTS AND A SPECTRAL SEQUENCE OF QUILLEN
}

\section{Christopher Allday}

\begin{abstract}
A third order rational Whitehead product is defined in terms of the appropriate differential graded Lie algebra. The product is used to calculate the second differential in Quillen's rational reverse Adams spectral sequence. Some facts about a fourth order product are stated, and conjectures are made concerning higher order products. The products of this paper are compared to those defined by Zeeman, Hardie, and Porter.
\end{abstract}

In the paper "Rational homotopy theory" [7], Quillen introduces a very interesting rational unstable reverse Adams spectral sequence. He also introduces a functor, which assigns to each one-connected topological space a differential graded Lie algebra, whose homology is the rational homotopy Lie algebra of the space. In this paper we show, first, how higher order rational Whitehead products may be defined using the $D G$ Lie algebra, secondly, how these Whitehead products may be used to calculate the differentials in Quillen's spectral sequence, and, thirdly, how these Whitehead products compare to those defined by Zeeman, Hardie, Porter, et al.

In the first section we review some of the notation and material of [7]. In the second section we define the third order rational Whitehead product, and show how it and the ordinary (second order) rational Whitehead product fit into Quillen's spectral sequence. Further, we indicate how the higher order products may be defined, but an explicit definition is included only for the fourth order product. In the final section a theorem is established, which provides a mechanism for comparing our rational products with those defined classically, and this comparison is carried out for the third order product.

1. Review of material and notation from [7]. We are concerned with the following three categories: $\mathscr{T}_{2}$, the category of 1-connected pointed topological spaces, and basepoint preserving continuous maps, whose weak equivalences are those maps which induce isomorphisms on rational homotopy.

$(D G L)_{1}$, the category of reduced differential graded Lie algebras orer $Q$ and $D G$ Lie algebra morphisms, whose weak equivalences are those morphisms which induce isomorphisms on homology.

$(D G C)_{2}$, the category of 2-reduced differential graded coalgebras over 
$Q$ and $D G$ coalgebra morphisms, whose weak equivalences are, again, those morphisms which induce isomorphisms on homology.

Quillen defines, implicitly, a functor

$$
\mu: \mathscr{T}_{2} \longrightarrow(D G L)_{1} \text {. }
$$

For a space $X$ in $\mathscr{F}_{2}$, the homology of $\mu(X)$ is the rational homotopy Lie algebra of $X, L_{*}(X)$. The latter may be obtained by setting $L_{n}(X)=\pi_{n+1}(X) \otimes Q$, and defining the Lie bracket of $\alpha \in L_{n}(X)$ and $\beta \in L_{m}(X)$ by the formula

$$
[\alpha, \beta]=(-1)^{n+1}[a, b] \otimes r s \in \pi_{n+m+1}(X) \otimes Q=L_{n+m}(X),
$$

where $r$ and $s$ are rational numbers such that $\alpha=a \otimes r$ and $\beta=$ $b \otimes s$, and where the bracket on the right is the conventional Whitehead product in $\pi_{*}(X)$. With this definition $L_{*}(X)$ is isomorphic to $\pi_{*}(\Omega X) \otimes Q$, endowed with the Samelson product.

Quillen also defines a functor

$$
\mathscr{C}:(D G L)_{1} \longrightarrow(D G C)_{2},
$$

which has the property that, for a space $X$ in $\mathscr{F}_{2}$, the homology of $\mathscr{C} \mu(X)$ is precisely the rational singular homology coalgebra of $X$.

For an object of $\mathscr{T}_{2}, X$, say, by considering the primitive filtration on $\mathscr{C} \mu(X)$, Quillen obtains a coalgebra spectral sequence,

$$
E^{1}=S\left(\Sigma L_{*}(X)\right) \Rightarrow H_{*}(X ; Q),
$$

where $\Sigma$ is the functor which raises the grading degree by one (e.g. $\left.\left(\Sigma L_{*}(X)\right)_{n}=L_{n-1}(X)\right)$, and $S$ is the symmetric algebra functor. The construction is functorial, and so the edge map

$$
\pi_{n}(X) \otimes Q \cong E_{1, n-1}^{1} \stackrel{\text { onto }}{\longrightarrow} E_{1, n-1}^{\infty} \stackrel{\subseteq}{\longrightarrow} H_{n}(X ; Q)
$$

is the rational Hurewicz homomorphism.

In order to establish the notation needed for the calculations of $\S 2$, below, it is necessary to review in detail Quillen's construction of the functor $\mathscr{C}$. To this end let $L$ be a reduced $D G$ Lie algebra, and define a $D G$ Lie algebra $\Sigma L \# L$. As a graded vector space $\Sigma L \# L$ is the direct sum of $\Sigma L$ and $L$. The inclusion maps $L \rightarrow$ $\Sigma L \rightarrow \Sigma L \# L$ and $L \rightarrow \Sigma L \# L$ are denoted by $\Sigma$ and $\theta$ respectively. (In addition, if $x$ is an element of degree $n$ in $L$, we shall denote by $\Sigma x$ the corresponding element of degree $n+1$ in $\Sigma L$.) The Lie bracket on $\Sigma L \# L$ is defined by requiring that, for all $x$ and $y$ in $L$,

(i) $[\Sigma x, \Sigma y]=0$

and

(ii) $[\Sigma x, \theta y]=\Sigma[x, y]$

(iii) $[\theta x, \theta y]=\theta[x, y]$. 
The differential on $\Sigma L \# L$ is defined by requiring that, for all $x$ in $L$, and

(i) $d \Sigma x=\theta x-\Sigma d^{\prime} x$,

(ii) $d \theta x=\theta d^{\prime} x$,

where $d^{\prime}$ is the differential on $L$. Letting $U$ denote the universal enveloping algebra functor, $\theta$ induces a right $U(L)$-module structure on $U(\Sigma L \# L) . \quad \mathscr{C}(L)$ is then the coalgebra $U(\Sigma L \# L) \otimes_{U(L)} Q$, and the differential on $\mathscr{C}$ is obtained by requiring that the natural surjection, $\pi: U(\Sigma L \# L) \rightarrow \mathscr{C}(L)$, be a $D G$ coalgebra morphism. If $i: S(\Sigma L) \rightarrow U(\Sigma L \# L)$ is the Hopf algebra map induced by the inclusion $\Sigma L \rightarrow \Sigma L \# L$, then $\pi i: S(\Sigma L) \rightarrow \mathscr{C}(L)$ is a coalgebra isomorphism.

2. Higher order Whitehead products and the spectral sequence. First we show how the ordinary (second order) Whitehead product determines the differential, $d^{1}$, of the spectral sequence.

THEOREM 2.1. Let $X$ be a one-connected pointed topological space, and let $a_{i}$ be an element of $L_{n_{i}}(x)$, for $i=1,2$. Then in Quillen's spectral sequence for $X$,

$$
d^{1}\left(\Sigma a_{1} \Sigma a_{2}\right)=(-1)^{n_{1}} \Sigma\left[a_{1}, a_{2}\right] .
$$

Proof. Let $\alpha_{i} \in \mu_{n_{i}}(x)$ be a cycle which represents $\alpha_{i}(i=1,2)$. Then in $U(\Sigma \mu(x) \# \mu(x))$ we have

$$
d\left(\Sigma \alpha_{1} \Sigma \alpha_{2}\right)=\theta \alpha_{1} \Sigma \alpha_{2}+(-1)^{n_{1}+1} \Sigma \alpha_{1} \theta \alpha_{2} .
$$

Now $\left[\theta \alpha_{1}, \Sigma \alpha_{2}\right]=\theta \alpha_{1} \Sigma \alpha_{2}-(-1)^{n_{1}\left(n_{2}+1\right)} \Sigma \alpha_{2} \theta \alpha_{1}$. Hence $\pi d\left(\Sigma \alpha_{1} \Sigma \alpha_{2}\right)=$ $\pi\left[\theta \alpha_{1}, \Sigma \alpha_{2}\right]=(-1)^{n_{1}} \pi \Sigma\left[\alpha_{1}, \alpha_{2}\right]$. I.e., where $d^{\prime}$ denotes the differential on $\mathscr{C} \mu(X)$,

$$
d^{\prime} \pi i\left(\Sigma \alpha_{1} \Sigma \alpha_{2}\right)=(-1)^{n_{1}} \pi i \Sigma\left[\alpha_{1}, \alpha_{2}\right] \text {. }
$$

The result now follows, because of the identifications made in constructing the spectral sequence.

Now suppose that $a_{i} \in L_{n_{i}}(X)$, for $i=1,2,3$, and that $\left[a_{2}, a_{3}\right]$, $\left[a_{3}, a_{1}\right]$, and $\left[a_{1}, a_{2}\right]$ are all zero. Let $\alpha_{i}$ be a cycle in $\mu_{n_{i}}(X)$, for $i=$ $1,2,3$, such that $\alpha_{i}$ represents $a_{i}$. For $1 \leqq i, j \leqq 3$, and $i \neq j$, let $\xi_{i j}$ be an element of $\mu_{n_{i}+n_{j}+1}(X)$, such that in $\mu(X), d \xi_{i j}=\left[\alpha_{i}, \alpha_{j}\right]$. Then by the Jacobi identity,

$$
(-1)^{n_{1} n_{3}}\left[\xi_{12}, \alpha_{3}\right]+(-1)^{n_{2} n_{1}}\left[\xi_{23}, \alpha_{1}\right]+(-1)^{n_{3} n_{2}}\left[\xi_{31}, \alpha_{2}\right]
$$

is a cycle in $\mu(X)$. Call this cycle $\left\langle\alpha_{1}, \alpha_{2}, \alpha_{3} ; \xi_{i j}\right\rangle$.

DEFINITION. With the notation and conditions of the above 
paragraph, we define the third order rational Whitehead product of $a_{1}, a_{2}, a_{3}$, denoted by $\left[a_{1}, a_{2}, a_{3}\right]$, to be the set of all elements of $L_{n_{1}+n_{2}+n_{3}+1}(X)$ represented by cycles of the form $\left\langle\alpha_{1}, \alpha_{2}, \alpha_{3} ; \xi_{i j}\right\rangle$.

Let $n_{1}+n_{2}+n_{3}=n$, and let $J_{n+1}\left(a_{1}, a_{2}, a_{3}\right)$ be the vector subspace of $L_{n+1}(X)$ spanned by all elements of the form $\left[a_{1}, x\right],\left[a_{2}, y\right]$, and $\left[a_{3}, z\right]$, for all $x \in L_{n+1-n_{1}}(X), y \in L_{n+1-n_{2}}(X)$, and $z \in L_{n+1-n_{3}}(X)$. The following proposition shows that $J_{n+1}\left(a_{1}, a_{2}, a_{3}\right)$ is the indeterminancy of $\left[a_{1}, a_{2}, a_{3}\right]$.

Proposition 2.2. With the above notation, $\left[a_{1}, a_{2}, a_{3}\right]$ is a coset of $J_{n+1}\left(a_{1}, a_{2}, a_{3}\right)$ in $L_{n+1}(X)$.

Proof. Let $a$ be an element of $\left[a_{1}, a_{2}, a_{3}\right] \subseteq L_{n+1}(X)$, and let $x \in$ $L_{n+1-n_{1}}(X)$. Suppose $a$ is represented by $\left\langle\alpha_{1}, \alpha_{2}, \alpha_{3} ; \xi_{i j}\right\rangle$, and let $\xi \epsilon$ $\mu_{n_{2}+n_{3}+1}(X)$ be a cycle representing $x$. Then $\left[\xi, \alpha_{1}\right]$ represents $\left[x, a_{1}\right]$, and $\left\langle\alpha_{1}, \alpha_{2}, \alpha_{3} ; \xi_{i j}\right\rangle+\left[\xi, \alpha_{1}\right]$ represents $a+\left[x, a_{1}\right]$. But $\left\langle a_{1}, a_{2}, a_{3} ; \xi_{i j}\right\rangle+$ $\left[\xi, \alpha_{1}\right]=\left\langle\alpha_{1}, \alpha_{2}, \alpha_{3} ; \xi_{i j}^{\prime}\right\rangle$, where $\xi_{12}^{\prime}=\xi_{12}, \xi_{23}^{\prime}=\xi_{23}+(-1)^{n_{2} n_{1} \xi}$, and $\xi_{31}^{\prime}=\xi_{31}$.

$\therefore \quad a+\left[x, a_{1}\right] \in\left[a_{1}, a_{2}, a_{3}\right]$.

$\therefore \quad a+J_{n+1}\left(a_{1}, a_{2}, a_{3}\right) \leqq\left[a_{1}, a_{2}, a_{3}\right]$.

Now let $b \in\left[a_{1}, a_{2}, a_{3}\right]$ be represented by a cycle $\left\langle a_{1}^{\prime}, a_{2}^{\prime}, a_{3}^{\prime} ; \xi_{i j}^{\prime}\right\rangle$. Since $\alpha_{i}^{\prime}$ represents $\alpha_{i}$, there must exist $\beta_{i} \in \mu_{n_{i}+1}(X)$, such that $\alpha_{i}^{\prime}=$ $\alpha_{i}+d \beta_{i}$. Suppose that $\beta_{2}$ and $\beta_{3}$ are zero. Then

$$
\begin{aligned}
d \xi_{12}^{\prime}= & {\left[\alpha_{1}^{\prime}, \alpha_{2}^{\prime}\right]=\left[\alpha_{1}, \alpha_{2}\right]+\left[d \beta_{1}, \alpha_{2}\right] } \\
d \xi_{31}^{\prime}= & {\left[\alpha_{3}^{\prime}, \alpha_{1}^{\prime}\right]=\left[\alpha_{3}, \alpha_{1}\right]+\left[\alpha_{3}, d \beta_{1}\right] } \\
d \xi_{23}^{\prime}= & {\left[\alpha_{2}^{\prime}, \alpha_{3}^{\prime}\right]=\left[\alpha_{2}, \alpha_{3}\right] } \\
\therefore & \xi_{12}-\xi_{12}^{\prime}+\left[\beta_{1}, \alpha_{2}\right], \\
& \xi_{31}-\xi_{31}^{\prime}+(-1)^{n_{3}}\left[\alpha_{3}, \beta_{1}\right],
\end{aligned}
$$

and $\xi_{23}-\xi_{23}^{\prime}$ are cycles. Call these cycles $\zeta_{12}, \zeta_{31}$, and $\zeta_{23}$, respectively. So

$$
\begin{aligned}
\left\langle\alpha_{1}^{\prime}, \alpha_{2}^{\prime}, \alpha_{3}^{\prime} ; \xi_{i j}^{\prime}\right\rangle= & \left\langle\alpha_{1}, \alpha_{2}, \alpha_{3} ; \xi_{i j}\right\rangle+(-1)^{n_{1} n_{3}}\left[\left[\beta_{1}, \alpha_{2}\right], \alpha_{3}\right] \\
& -(-1)^{n_{1} n_{3}}\left[\zeta_{12}, \alpha_{3}\right]+(-1)^{n_{2} n_{1}}\left[\xi_{23}, d \beta_{1}\right]-(-1)^{n_{2} n_{1}}\left[\zeta_{23}, \alpha_{1}^{\prime}\right] \\
& +(-1)^{n_{3} n_{2}+n_{3}}\left[\left[\alpha_{3}, \beta_{1}\right], \alpha_{2}\right]-(-1)^{n_{3} n_{2}}\left[\zeta_{31}, \alpha_{2}\right] \\
= & \left\langle\alpha_{1}, \alpha_{2}, \alpha_{3} ; \xi_{i j}\right\rangle-(-1)^{n_{1} n_{3}}\left[\zeta_{12}, \alpha_{3}\right] \\
& -(-1)^{n_{3} n_{2}}\left[\zeta_{31}, \alpha_{2}\right]-(-1)^{n_{1} n_{2}}\left[\zeta_{23}, \alpha_{1}^{\prime}\right] \\
& +(-1)^{n_{2}+n_{3}+n_{1} n_{2}+1} d\left[\xi_{23}, \beta_{1}\right] .
\end{aligned}
$$

Let the homology classes of $\zeta_{23}, \zeta_{31}$, and $\zeta_{12}$ be $x, y$, and $z$, respectively. Then

$$
b=a-(-1)^{n_{1} n_{3}}\left[z, a_{3}\right]-(-1)^{n_{3} n_{2}}\left[y, a_{2}\right]-(-1)^{n_{1} n_{2}}\left[x, a_{1}\right]
$$


Hence, for all $a, b \in\left[a_{1}, a_{2}, a_{3}\right], a-b \in J_{n+1}\left(a_{1}, a_{2}, a_{3}\right)$.

In the following, if $\left\{E_{i j}^{r}\right\}$ is the Quillen spectral sequence of some space, and if $x$ is an element of $E_{i j}^{r}$ such that $d^{r}(x)=0$, then we shall let $[x]^{(r+1)}$ denote the class in $E_{i j}^{r+1}$ which is represented by $x$.

CoROLlaRY 2.3. Let $X$ be a space of $\mathscr{T}_{2}$, let $a_{i} \in L_{n_{i}}(X), i=$ $1,2,3$, be such that $\left[a_{2}, a_{3}\right],\left[a_{3}, a_{1}\right]$, and $\left[a_{1}, a_{2}\right]$ are all zero. Let $a, b \in$ $\left[a_{1}, a_{2}, a_{3}\right] \subseteq L_{n+1}(X)$, where $n=n_{1}+n_{2}+n_{3}$. Then in the Quillen spectral sequence of $X,[\Sigma a]^{(2)}=[\Sigma b]^{(2)}$ in $E_{1, n+1}^{2}$.

Theorem 2.4. With $X, a_{1}, a_{2}, a_{3}$ and $a$ as in the above corollary, then in the Quillen spectral sequence of $X, d^{1}\left(\Sigma a_{1} \Sigma a_{2} \Sigma a_{3}\right)=0$, and

$$
d^{2}\left[\Sigma a_{1} \Sigma a_{2} \Sigma a_{3}\right]^{(2)}=(-1)^{n_{1} n_{3}+n_{2}+1}[\Sigma a]^{(2)} \text {. }
$$

Proof. We calculate as in the proof of Theorem 2.1. Let $\alpha_{i} \in \mu_{n_{i}}(X)$ be a cycle which represents $a_{i}(i=1,2,3)$. Then in $U(\Sigma \mu(X) \# \mu(X))$ we have

$$
\begin{aligned}
& d\left(\Sigma \alpha_{1} \Sigma \alpha_{1} \Sigma \alpha_{3}\right)=d\left(\Sigma \alpha_{1} \Sigma \alpha_{2}\right) \Sigma \alpha_{3}+(-1)^{n_{1}+n_{2}} \sum \alpha_{1} \Sigma \alpha_{2} d\left(\sum \alpha_{3}\right) \\
& =\theta \alpha_{1} \Sigma \alpha_{2} \Sigma \alpha_{3}+(-1)^{n_{1}+1} \Sigma \alpha_{1} \theta \alpha_{2} \Sigma \alpha_{3}+(-1)^{n_{1}+n_{2}} \sum \alpha_{1} \Sigma \alpha_{2} \Sigma \alpha_{3} \\
& \therefore \quad \pi d\left(\Sigma \alpha_{1} \Sigma \alpha_{2} \Sigma \alpha_{3}\right)=\pi\left(\left[\theta \alpha_{1}, \Sigma \alpha_{2}\right] \Sigma \alpha_{3}+(-1)^{n_{1}\left(n_{2}+1\right)} \Sigma \alpha_{2} \Sigma \alpha_{1} \Sigma \alpha_{3}\right. \\
& \left.+(-1)^{n_{1}+1} \Sigma \alpha_{1}\left[\theta \alpha_{2}, \Sigma \alpha_{3}\right]\right) \\
& =\pi\left((-1)^{n_{1}} \Sigma\left[\alpha_{1}, \alpha_{2}\right] \Sigma \alpha_{3}+(-1)^{n_{1} n_{2}} \Sigma \alpha_{2} \Sigma\left[\alpha_{1}, \alpha_{3}\right]\right. \\
& \left.+(-1)^{n_{1}+n_{2}+1} \Sigma \alpha_{1} \Sigma\left[\alpha_{2}, \alpha_{3}\right]\right) \text {. }
\end{aligned}
$$

Now

$$
\begin{aligned}
\Sigma\left[\alpha_{1}, \alpha_{j}\right] \Sigma \alpha_{k} & =\Sigma d \xi_{i j} \Sigma \alpha_{k} \\
& =\theta \xi_{i j} \Sigma \alpha_{k}-d\left(\Sigma \xi_{i j}\right) \Sigma \alpha_{k} \\
& =\theta \xi_{i j} \Sigma \alpha_{k}-d\left(\Sigma \xi_{i j} \Sigma \alpha_{k}\right)+(-1)^{n_{i}+n_{j}+1} \Sigma \xi_{i j} d \Sigma \alpha_{k} \\
& =\theta \xi_{i j} \Sigma \alpha_{k}-d\left(\Sigma \xi_{i j} \Sigma \alpha_{k}\right)+(-1)^{n_{i}+n_{j}+1} \Sigma \xi_{i j} \theta \alpha_{k}
\end{aligned}
$$

and

$$
\begin{aligned}
\Sigma \alpha_{i} \Sigma\left[\alpha_{j}, \alpha_{k}\right] & =\Sigma \alpha_{i} \theta \xi_{j k}-\Sigma \alpha_{i} d\left(\Sigma \xi_{j k}\right) \\
& =\Sigma \alpha_{i} \theta_{j k}+(-1)^{n_{i}} d\left(\Sigma \alpha_{i} \Sigma \xi_{j k}\right)+(-1)^{n_{i}+1} \theta \alpha_{i} \Sigma \xi_{j k}
\end{aligned}
$$

where $\xi_{i j}$ is such that $d \xi_{i j}=\left[\alpha_{i}, \alpha_{j}\right]$. Again let $d^{\prime}$ be the differential in $\mathscr{C} \mu(X)$. Then

$$
\begin{aligned}
d^{\prime} \pi i\left(\Sigma \alpha_{1} \Sigma \alpha_{2} \Sigma \alpha_{3}\right)= & \pi i\left((-1)^{n_{2}+1} \Sigma\left[\xi_{12}, \alpha_{3}\right]\right. \\
& \left.+(-1)^{n_{1} n_{2}+1} \Sigma\left[\alpha_{2}, \xi_{13}\right]+(-1)^{n_{1} n_{2}} \Sigma\left[\alpha_{1}, \xi_{23}\right]\right)
\end{aligned}
$$




$$
\begin{aligned}
& +d^{\prime} \pi i\left((-1)^{n_{1}+1} \Sigma \xi_{12} \Sigma \alpha_{3}+(-1)^{n_{1} n_{2}+n_{2}} \Sigma \alpha_{2} \Sigma \xi_{1_{3}}\right. \\
& \left.+(-1)^{n_{2}+1} \Sigma \alpha_{1} \Sigma \xi_{23}\right) \\
= & \pi i\left((-1)^{n_{2}+1} \Sigma\left[\xi_{12}, \alpha_{3}\right]+(-1)^{n_{1} n_{3}+n_{2} n_{3}+n_{2}+1} \Sigma\left[\xi_{31}, \alpha_{2}\right]\right. \\
& \left.+(-1)^{n_{1} n_{2}+n_{1} n_{3}+n_{2}+1} \Sigma\left[\xi_{23}, \alpha_{1}\right]\right)+d^{\prime} \pi i\left((-1)^{n_{1}+1} \Sigma \xi_{12} \Sigma \alpha_{3}\right. \\
& +(-1)^{n_{1} n_{3}+n_{2} n_{3}+n_{1}+n_{2}+n_{3}+1} \Sigma \xi_{31} \Sigma \alpha_{2}^{\prime} \\
& \left.+(-1)^{n_{1} n_{2}+n_{1} n_{3}+n_{3}+1} \Sigma \xi_{23} \Sigma \alpha_{1}\right) \\
= & (-1)^{n_{1} n_{3}+n_{2}+1} \pi i \Sigma\left\langle\alpha_{1}, \alpha_{2}, \alpha_{3}^{\prime} ; \xi_{i j}\right\rangle+d^{\prime} \pi i
\end{aligned}
$$

where

$$
\begin{aligned}
\zeta= & (-1)^{n_{1}+1} \sum \xi_{12} \Sigma \alpha_{3}+(-1)^{n_{1} n_{3}+n_{2} n_{3}+n_{1}+n_{2}+n_{3}+1} \sum \xi_{31} \Sigma \alpha_{2} \\
& +(-1)^{n_{1} n_{2}+n_{1} n_{3}+n_{3}+1} \sum \xi_{23} \Sigma \alpha_{1} .
\end{aligned}
$$

Again the result follows because of the identifications made in constructing the spectral sequence. In particular, the term $d^{\prime} \pi i s$ is explained away by formula (3.8) of MacLane [3], Chapter XI, §3, applied to $E_{3, n}^{2}$.

REMARK 1. It is clear from the definition that the third order product has the usual functoriality, and the usual trilinearity but different change of sign under permutation.

Specifically, if $X$ and $Y$ are spaces of $\mathscr{T}_{2}$, if $f: X \rightarrow Y$ is a basepoint preserving map, and if $\left[a_{1}, a_{2}, a_{3}\right]$ is a well defined third order product in $L_{*}(X)$, then $\left[f_{*}\left(a_{1}\right), f_{*}\left(a_{2}\right), f_{*}\left(a_{3}\right)\right]$ is well defined in $L_{*}(Y)$, and

$$
f_{*}\left(\left[a_{1} a_{2} a_{3}\right]\right) \subseteq\left[f_{*}\left(a_{1}\right), f_{*}\left(a_{2}\right), f_{*}\left(a_{3}\right)\right] \text {. }
$$

Also if $\left[\alpha_{1}, a_{2}, a_{3}\right]$ and $\left[\alpha_{1}, a_{2}, \alpha_{3}^{\prime}\right]$ are both well defined, then so is $\left[a_{1}, a_{2}, a_{3}+a_{3}^{\prime}\right]$, and

$$
\left[a_{1}, a_{2}, a_{3}+a_{3}^{\prime}\right] \subseteq\left[a_{1}, a_{2}, a_{3}\right]+\left[a_{1}, a_{2}, a_{3}^{\prime}\right] .
$$

Similarly for the other variables.

However, $\left[a_{1}, a_{2}, a_{3}\right]=(-1)^{n_{1} n_{2}+n_{1} n_{3}+n_{2} n_{3}+1}\left[a_{2}, a_{1}, a_{3}\right]=\left[a_{2}, a_{3}, a_{1}\right]$, and so on. (Cf. Hardie [2].)

REMARK 2. The general definition for $k$ th order products seems hard to calculate. For example, let $X$ be a space in $\mathscr{T}_{2}$, let $a_{i} \in$ $L_{n_{i}}(X), i=1,2,3,4$, be elements such that

and

(i) $i \neq j \Rightarrow\left[a_{i}, a_{j}\right]=0$,

(ii) $i, j, k$ all distinct $\Rightarrow 0 \in\left[a_{i}, a_{j}, a_{k}\right]$.

Let $\xi_{i j}, i \neq j$, and $\xi_{i j k}, i, j, k$ all distinct, be such that

(i) $d \xi_{i j}=\left[\alpha_{i}, \alpha_{j}\right]$ 
and

(ii) $d \xi_{i j k}=\left\langle\alpha_{i}, \alpha_{j}, \alpha_{k} ; \xi_{i j}\right\rangle$, where $\alpha_{i}$ is a cycle in $\mu_{n_{i}}(X)$, representing $a_{i}, i=1,2,3,4$. Then a calculation similar to the above yields the following result:

$$
d^{3}\left[\Sigma a_{1} \Sigma a_{2} \Sigma a_{3} \Sigma a_{4}\right]^{(3)}=[\Sigma \alpha]^{(3)},
$$

where $a \in L_{n_{1}+n_{2}+n_{3}+n_{4}+2}(X)$ is represented by the cycle

$$
\begin{aligned}
& (-1)^{n_{1} n_{3}+n_{1}+n_{3}+1}\left[\xi_{123}, \alpha_{4}\right]+(-1)^{n_{1} n_{4}+n_{3} n_{4}+n_{1}+n_{3}}\left[\xi_{124}, \alpha_{3}\right] \\
+ & (-1)^{n_{1} n_{4}+n_{2} n_{3}+n_{2} n_{4}+n_{1}+n_{3}+1}\left[\xi_{134}, \alpha_{2}\right]+(-1)^{n_{1} n_{2}+n_{1} n_{3}+n_{1} n_{4}+n_{2} n_{4}+n_{1}+n_{3}}\left[\xi_{234}, \alpha_{1}\right] \\
+ & (-1)^{n_{2}+n_{3}+1}\left[\xi_{12}, \xi_{34}\right]+(-1)^{n_{2} n_{3}}\left[\xi_{13}, \xi_{24}\right]+(-1)^{n_{2} n_{4}+n_{3} n_{4}+n_{3}+n_{4}+1}\left[\xi_{14}, \xi_{23}\right] .
\end{aligned}
$$

Before stating a conjecture for the general form of the $k$ th order product, we recall from May [4], for example, that if $p+q=k$, then $(\mu, \nu)$ is a $(p, q)$-shuffle if $\mu(i)=\pi(i)$ for $1 \leqq i \leqq p$ and $\nu(j)=\pi(p+j)$ for $1 \leqq j \leqq q$, where $\pi$ is a permutation of $\{1, \cdots, k\}$ such that $\pi(i)<$ $\pi(j)$ for $1 \leqq i<j \leqq p$ or for $p+1 \leqq i<j \leqq k$. $(\mu, \nu)$ is a $(p, q)$ shuffle of type II relative to 1 , if $\mu(1)=1$. Now suppose that $x_{i} \in$ $L_{n_{i}}(X), i=1, \cdots, k$, are elements such that $1 \leqq i_{1}<\cdots<i_{r} \leqq k$ and $2 \leqq r \leqq k-1$ imply that $\left[x_{i_{1}}, \cdots, x_{i_{r}}\right]$ is defined and includes zero. Let $\xi_{i}$ be a cycle in $\mu_{n_{i}}(X)$ which represents $x_{i}, 1 \leqq i \leqq k$, and for $1 \leqq i_{1}<\cdots<i_{r} \leqq k$ and $2 \leqq r \leqq k-1$, let $\xi_{i_{1} \cdots i_{r}}$ be an element of $\mu(X)$, such that $d \xi_{i_{1} \cdots i_{r}}$ represents the element of $\left[x_{i_{1}}, \cdots, x_{i_{r}}\right]$, represented by the cycle $\left\langle\xi_{i_{1}}, \xi_{i_{1} i_{2}}, \cdots, \xi_{i_{1} \cdots i_{r-1}}\right\rangle$ constructed recursively as follows.

For $k=2,\left\langle\xi_{i_{1}}\right\rangle=(-1)^{n_{1}}\left[\xi_{1}, \xi_{2}\right]$.

For $k=3,\left\langle\xi_{i_{1}}, \xi_{i_{1} i_{2}}\right\rangle=(-1)^{n_{1} n_{3}+n_{2}+1}\left\langle\xi_{1}, \xi_{2}, \xi_{3} ; \xi_{i_{1} i_{2}}\right\rangle$, as above.

$$
\left\langle\xi_{i_{1}}, \xi_{i_{1} i_{2}}, \cdots, \xi_{i_{1} \cdots i_{k-1}}\right\rangle=\sum_{p=1}^{k-1} \sum \pm\left[\xi_{\mu}, \xi_{2}\right],
$$

where the second sum is taken over all $(p, q)$-shuffles, $(\mu, \nu)$, of type II relative to 1 , with $p+q=k$, and where $\xi_{\mu}=\xi_{\mu(1) \ldots \mu(p)}$ and $\xi_{\nu}=$ $\xi_{\nu(1) \ldots \nu(q)}$. The conjecture is that the signs of the terms, $\left[\xi_{\mu}, \xi_{\nu}\right]$, can be chosen to make $\left\langle\xi_{i_{1}}, \xi_{i_{1} i_{2}}, \cdots, \xi_{i_{1} \cdots i_{k-1}}\right\rangle$ into a cycle, representing an element $x$, say, of $L_{n+k-2}(X)$, where $n=\sum_{i=1}^{k} n_{i}$; and that such elements define the $k$ th order product $\left[x_{1}, \cdots, x_{k}\right]$. Furthermore, we make the conjecture that if $x$ is such an element of $\left[x_{1}, \cdots, x_{k}\right]$, then in the Quillen spectral sequence for $X,\left[\Sigma x_{1} \cdots \Sigma x_{k}\right]^{(k-1)}$ exists, and

$$
d^{k-1}\left[\Sigma x_{1} \cdots \Sigma x_{k}\right]^{(k-1)}=[\Sigma x]^{(k-1)} .
$$

3. Comparison with the classical definition. We recall from Hardie [2], Porter [6], or more specifically, Arkowitz [1], the following 
definition of a $k$ th order Whitehead product in ordinary homotopy. Let $X$ be a space in $\mathscr{T}_{2}$, and let $a_{i} \in \pi_{n_{i}+1}, i=1, \cdots, k$, be homotopy classes. Let $P$ be the product, $S^{n_{1}+1} \times \cdots \times S^{n_{k}+1}$, let $S$ be the (lean) wedge, $S^{n_{1}+1} \vee \cdots \vee S^{n_{k}+1}$, and let $T$ be the fat wedge, $T\left(S^{n_{1}+1}, \cdots, S^{n_{k}+1}\right)$, consisting of $k$-tuples from $P$, which have at least one coordinate at the base point. Let $g: S \rightarrow X$ be the map induced by the $a_{i}$. That is, we choose a particular representative $f_{i}: S^{n_{i}+1} \rightarrow X, i=1, \cdots, k$, for each $a_{i}$; and $g$ is the unique map such that $g \circ i n_{i}=f_{i}, i=1, \cdots, k$, where $i n_{i}: S^{n_{i}+1} \rightarrow S$ is the natural inclusion. Let $n=\sum_{i=1}^{k} n_{i}$, and choose a generator $z \in H_{n+k}(P, T)$. Suppose there exists an extension of $g, \hat{g}: T \rightarrow X$. Then we have a diagram

$$
H_{n+k}(P, T) \stackrel{h}{\longleftarrow} \pi_{n+k}(P, T) \stackrel{\partial}{\longrightarrow} \pi_{n+k-1}(T) \stackrel{\hat{g}_{\sharp}}{\longrightarrow} \pi_{n+k-1}(X),
$$

where the Hurewicz map $h$ is an isomorphism. The $k$ th order Whitehead product, $\left[a_{1}, \cdots, a_{k}\right]$, is defined to be $\left\{\hat{g}_{\#} \partial h^{-1}(z): \hat{g}\right.$ is an extension of $g\} \subseteq \pi_{n+k-1}(X)$. If no extensions exist, then $\left[a_{1}, \cdots, a_{k}\right]$ is empty.

In order to obtain $k$ th order Whitehead products in rational homotopy, we repeat the above construction after localizing the spaces concerned at the empty set of primes. (Here we follow a suggestion of the referee.) This localization will be denoted, as usual, by the subscript zero. Thus for $a_{i} \in L_{n_{i}}(X) \cong \pi_{n_{i}+1}\left(X_{0}\right), i=1, \cdots, k$, we obtain a map $g_{o}: S_{o} \rightarrow X_{o}$, and define the $k$ th order rational Whitehead product, $\left[a_{1}, \cdots, a_{k}\right]^{\prime}$, to be $\left\{\hat{g}_{0 \ddagger} \partial h^{-1}\left(z_{0}\right): \hat{g}_{0}\right.$ is an extension of $g_{0}$ to $\left.T_{0}\right\}$. The element $z_{0}$ is just the image of $z$, above, under the localization map, and $\partial h^{-1}$ comes from the diagram

$$
H_{n+k}\left(P_{0}, T_{0}\right) \stackrel{h}{\longleftarrow} \pi_{n+k}\left(P_{0}, T_{0}\right) \stackrel{\partial}{\longrightarrow} \pi_{n+k-1}\left(T_{0}\right) .
$$

We have, then, $\left[a_{1}, \cdots, a_{k}\right]^{\prime} \cong \pi_{n+k-1}\left(X_{0}\right) \cong L_{n+k-2}(X)$.

Throughout the following, we shall assume that $\min \left\{n_{i}: 1 \leqq i \leqq k\right\} \geqq$ 1 , so that $S, T$, and $P$ are all one-connected. We shall assume also that $k \geqq 2$.

The following lemma shows that the classical rational Whitehead products are not trivial.

LEMMA 3.1. With the above notation,

$$
\partial h^{-1}\left(z_{0}\right) \neq 0 \in \pi_{n+k-1}\left(T_{0}\right) \text {. }
$$

Proof. The inclusion, $S \rightarrow P$, induces an epimorphism, $\pi_{n}(S) \rightarrow$ $\pi_{n}(P)$, for all $n$. Hence the inclusion, $T \rightarrow P$, induces an epimorphism, $\pi_{n}(T) \rightarrow \pi_{n}(P)$, for all $n$. The map, $\partial: \pi_{n+k}(P, T) \rightarrow \pi_{n+k-1}(T)$, is, therefore a monomorphism. $\pi_{n+k}(P, T) \cong H_{n+k}(P, T) \cong Z$, and the result follows. 
The next proposition will be useful for analyzing the Quillen spectral sequence of $P$, and hence of $T$, too.

Proposition 3.2. Let $X_{1}, \cdots, X_{n}$ be spaces of $\mathscr{T}_{2}$. Then the Quillen spectral sequence of $X_{1} \times \cdots \times X_{n}, E^{r}\left(X_{1} \times \cdots \times X_{n}\right)$, say, is the tensor product of the spectral sequences of $X_{1}, \cdots, X_{n}, E^{r}\left(X_{1}\right), \cdots$, $E^{r}\left(X_{n}\right)$, say. That is, for all $r \geqq 1$, there is a DG-coalgebra isomorphism

$$
E^{r}\left(X_{1} \times \cdots \times X_{n}\right) \cong E^{r}\left(X_{1}\right) \otimes \cdots \otimes E^{r}\left(X_{n}\right) .
$$

Proof. Using induction it is clearly enough to show the result for the product of two spaces, $X$ and $Y$, say.

For $D G$ Lie algebras, $L$ and $L^{\prime}$, let $L \times L^{\prime}$ denote their product. We recall that as a vector space $L \times L^{\prime}$ is just $L \oplus L^{\prime}$, and the differential and Lie bracket are given by the obvious formulae:

$$
\begin{aligned}
d\left(x, x^{\prime}\right) & =\left(d x, d^{\prime} x^{\prime}\right), \\
{\left[\left(x, x^{\prime}\right),\left(y, y^{\prime}\right)\right] } & =\left([x, y],\left[x^{\prime}, y^{\prime}\right]\right) .
\end{aligned}
$$

This is clearly a product in the category $(D G L)_{1}$.

First we show that there exists a weak equivalence

$$
\theta: \mu(X \times Y) \longrightarrow \mu(X) \times \mu(Y) \text {. }
$$

Let $p_{1}, p_{2}$ be the projections of $X \times Y$ onto $X, Y$ respectively. Then we have a diagram in $(D G L)_{1}$ :

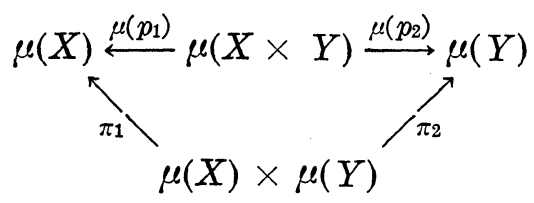

where $\pi_{1}$ and $\pi_{2}$ are the projections. Then there exists a unique

$$
\theta: \mu(X \times Y) \longrightarrow \mu(X) \times \mu(Y)
$$

making the diagram commute. To see that $\theta$ is a weak equivalence, take homology. We then have the commutative diagram:

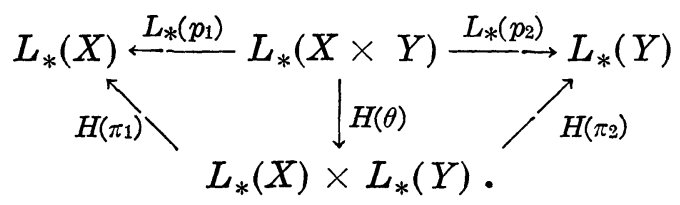

$H(\theta)$ must be an isomorphism, since it is unique in making the diagram commute, and it is known from elementary homotopy theory that there is an isomorphism, which makes this diagram commute. 
For $D G$ coalgebras $A$ and $B$, denote their product by $A \Pi B$. The functor $\mathscr{C}:(D G L)_{1} \rightarrow(D G C)_{2}$ is a right adjoint, and so preserves products. (Pareigis [5].) Also $\mathscr{C}$ carries weak equivalences into weak equivalences. Hence there is a weak equivalence in $(D G C)_{2}$,

$$
\mathscr{C}(\theta): \mathscr{C} \mu(X \times Y) \longrightarrow \mathscr{C} \mu(X) \Pi \mathscr{C} \mu(Y) \text {. }
$$

As a $D G$ coalgebra, $\mathscr{C} \mu(X) \Pi \mathscr{C} \mu(Y) \cong \mathscr{C} \mu(X) \otimes \mathscr{C} \mu(Y)$; and, where $F_{m}, m \geqq 0$, denotes the primitive filtration, it is clear that

$$
F_{m}(\mathscr{C} \mu(X) \otimes \mathscr{C} \mu(Y))=\sum_{p+q=m} F_{p}(\mathscr{C} \mu(X)) \otimes F_{q}(\mathscr{C} \mu(Y)) .
$$

Then $\mathscr{C}(\theta)$ induces a weak equivalence

$$
\mathscr{C}(\theta)^{\circ}: E^{\circ}(X \times Y) \longrightarrow E^{\circ}(X) \otimes E^{\circ}(Y),
$$

and, hence, $\mathscr{C}(\theta)$ induces isomorphisms

$$
\mathscr{C}(\theta)^{r}: E^{r}(X \times Y) \longrightarrow E^{r}(X) \otimes E^{r}(Y)
$$

for $r \geqq 1$.

CoRollary 3.3. In the Quillen spectral sequence of $P$, the differential $d^{r}$ is trivial for $r \geqq 2$.

Proof. The Quillen spectral sequence is trivial for odd dimensional spheres, and has $d^{1}$ as the only nontrivial differential for even dimensional spheres.

In the following theorem we shall continue to use the above notation. Let $a_{i} \in L_{n_{i}}(T)$ be the homotopy classes induced by the inclusions, $S^{n_{i}+1} \rightarrow T, i=1, \cdots, k$, and let

$$
a=\partial h^{-1}\left(z_{0}\right) \in \pi_{n+k-1}\left(T_{0}\right) \cong L_{n+k-2}(T) \text {. }
$$

Proposition 3.4. In the Quillen spectral sequence for $T$, $\left[\Sigma a_{1} \cdots \Sigma a_{k}\right]^{(k-1)}$ exists, $[\Sigma a]^{(k-1)}$ is nonzero, and there is a nonzero rational number $\alpha$, such that

$$
d^{k-1}\left[\Sigma a_{1} \cdots \Sigma a_{k}\right]^{(k-1)}=\alpha[\Sigma a]^{(k-1)} .
$$

Proof. We compare the spectral sequence of $T$ with that of $P$ via the inclusion map $i: T \rightarrow P$. For $r \geqq 1, i$ induces an isomorphism on all terms generated by $\sum_{m=1}^{n+k-3} E_{1, m}^{1}(T)$. It is clear then, that and

(i) $d^{r}\left[\Sigma a_{1} \cdots \Sigma a_{k}\right]^{(r)}=0$ for $1 \leqq r<k-1$,

(ii) $[\Sigma a]^{(k-1)} \neq 0$. 
Furthermore, if $[\Sigma a]^{(k-1)}$ and $\left[\Sigma a_{1} \cdots \Sigma a_{k}\right]^{(k-1)}$ survive to $E^{k}(T)$, they must survive to $E^{\infty}(T)$, since there are no terms to kill them. But killed they must be, since $\operatorname{dim} T \leqq n+k-2$. The result follows.

Now let $X$ be a space in $\mathscr{T}_{2}$, and let $x_{i} \in \pi_{n_{i+1}}(X), i=1,2,3$, be homotopy classes such that $\left[x_{2}, x_{3}\right],\left[x_{3}, x_{1}\right]$ and $\left[x_{1}, x_{2}\right]$ are all of finite order. Let $a_{i}=x_{i} \otimes 1 \in L_{n_{i}}(X), i=1,2,3$, be the corresponding rational classes, and so $\left[a_{1}, a_{2}, a_{3}\right]^{\prime}$ is the classical third order rational product. The indeterminacy of $\left[a_{1}, a_{2}, a_{3}\right]^{\prime}$ is $J_{n+1}\left(a_{1}, a_{2}, a_{3}\right)$, the same as for $\left[a_{1}, a_{2}, a_{3}\right]$. (Hardie [2].) We have the following corollary of Proposition 3.4.

CoROLlary 3.5. For $a \in\left[a_{1}, a_{2}, a_{3}\right]$ and $b \in\left[a_{1}, a_{2}, a_{3}\right]^{\prime}$, there is a nonzero rational number $\alpha$, such that $a-\alpha b$ is a sum of rational (second order) Whitehead products in $L_{n+1}(X)$.

Proof. Let $\hat{g}_{0}$ be an extension of $g_{0}: S_{0} \rightarrow X_{0}$ to $T_{0}$, where $g: S \rightarrow X$ is determined by $x_{i}, i=1,2,3$. Then, if $\partial h^{-1}\left(z_{0}\right)=c, \widehat{g}_{0 \ddagger}(c)$ is a representative of $\left[a_{1}, a_{2}, a_{3}\right]^{\prime}$. Thus $b-\hat{g}_{\text {o\# }}(c)$ is a sum of rational Whitehead products.

Now, from Proposition 3.4,

$$
d^{2}\left[\Sigma a_{1} \Sigma a_{2} \Sigma a_{3}\right]^{(2)}=\beta\left[\Sigma \hat{g}_{0 \sharp}(c)\right]^{(2)},
$$

where $\beta$ is a nonzero rational number.

But, from Theorem 2.4,

$$
d^{2}\left[\Sigma a_{1} \Sigma a_{2} \Sigma a_{3}\right]^{(2)}=(-1)^{n_{1} n_{3}+n_{2}+1}[\Sigma a]^{(2)} \text {. }
$$

Thus $(-1)^{n_{1} n_{3}+n_{2}+1} a-\beta \widehat{g}_{0 \sharp}(c)$ is a sum of rational Whitehead products by Theorem 2.1, and the result follows.

\section{REFERENCES}

1. M. Arkowitz, Whitehead products as images of Pontrjagin products, Trans. Amer. Math. Soc., 158 (1971), 453-463.

2. K. A. Hardie, On a construction of E. C. Zeeman, J. London Math. Soc., 35 (1960), 452-464.

3. S. MacLane, Homology, Springer-Verlag, Berlin, 1963.

4. J. P. May, Simplicial Objects in Algebraic Topology, Van Nostrand, Princeton, 1967.

5. B. Pareigis, Categories and Functors, Academic Press, New York, 1970.

6. G. J. Porter, Higher order Whitehead products, Topology, 3 (1965), 123-135.

7. D. G. Quillen, Rational homotopy theory, Ann. of Math., 90 (1969), 205-295.

Received March 16, 1972.

UNIVERSITY OF HAWAII 



\section{PACIFIC JOURNAL OF MATHEMATICS}

\section{EDITORS}

D. Gilbarg and J. Milgram

Stanford University

Stanford, California 94305

\author{
R. A. Beaumont \\ University of Washington \\ Seattle, Washington 98105
}

J. DuGUNDJI

Department of Mathematics

University of Southern California

Los Angeles, California 90007

Richard ARENS

University of California

Los Angeles, California 90024

\section{ASSOCIATE EDITORS}
E. F. BECKENBACH
B. H. NeumanN
F. WOLF
K. YosHIDA

\section{SUPPORTING INSTITUTIONS}

UNIVERSITY OF BRITISH COLUMBIA

CALIFORNIA INSTITUTE OF TECHNOLOGY

UNIVERSITY OF CALIFORNIA

MONTANA STATE UNIVERSITY

UNIVERSITY OF NEVADA

NEW MEXICO STATE UNIVERSITY

OREGON STATE UNIVERSITY

UNIVERSITY OF OREGON

OSAKA UNIVERSITY

\author{
UNIVERSITY OF SOUTHERN CALIFORNIA \\ STANFORD UNIVERSITY \\ UNIVERSITY OF TOKYO \\ UNIVERSITY OF UTAH \\ WASHINGTON STATE UNIVERSITY \\ UNIVERSITY OF WASHINGTON
* * * *
AMERICAN MATHEMATICAL SOCIETY \\ NAVAL WEAPONS CENTER
}

The Supporting Institutions listed above contribute to the cost of publication of this Journal, but they are not owners or publishers and have no responsibility for its content or policies.

Mathematical papers intended for publication in the Pacific Journal of Mathematics should be in typed form or offset-reproduced, (not dittoed), double spaced with large margins. Underline Greek letters in red, German in green, and script in blue. The first paragraph or two must be capable of being used separately as a synopsis of the entire paper. The editorial "we" must not be used in the synopsis, and items of the bibliography should not be cited there unless absolutely necessary, in which case they must be identified by author and Journal, rather than by item number. Manuscripts, in duplicate if possible, may be sent to any one of the four editors. Please classify according to the scheme of Math. Rev. Index to Vol. 39. All other communications to the editors should be addressed to the managing editor, Richard Arens, University of California, Los Angeles, California, 90024.

50 reprints are provided free for each article; additional copies may be obtained at cost in multiples of 50 .

The Pacific Journal of Mathematics is issued monthly as of January 1966. Regular subscription rate: $\$ 48.00$ a year (6 Vols., 12 issues). Special rate: $\$ 24.00$ a year to individual members of supporting institutions.

Subscriptions, orders for back numbers, and changes of address should be sent to Pacific Journal of Mathematics, 103 Highland Boulevard, Berkeley, California, 94708.

PUBLISHED BY PACIFIC JOURNAL OF MATHEMATICS, A NON-PROFIT CORPORATION

Printed at Kokusai Bunken Insatsusha (International Academic Printing Co., Ltd.), 270, 3-chome Totsuka-cho, Shinjuku-ku, Tokyo 160, Japan. 


\section{Pacific Journal of Mathematics}

\section{Vol. 46, No. 2 December, 1973}

Christopher Allday, Rational Whitehead products and a spectral sequence of

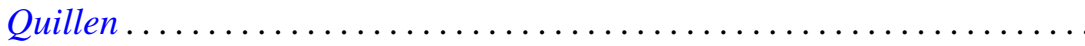

James Edward Arnold, Jr., Attaching Hurewicz fibrations with fiber

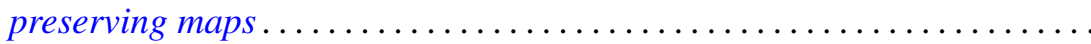

Catherine Bandle and Moshe Marcus, Radial averaging transformations with various metrics.................................

David Wilmot Barnette, A proof of the lower bound conjecture for convex

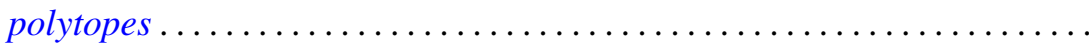

Louis Harvey Blake, Simple extensions of measures and the preservation of

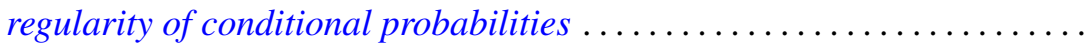

James W. Cannon, New proofs of Bing's approximation theorems for

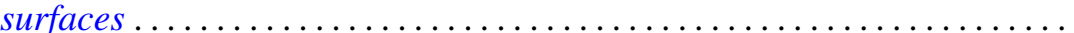

C. D. Feustel and Robert John Gregorac, On realizing HNN groups in

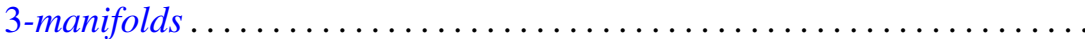

Theodore William Gamelin, Iversen's theorem and fiber algebras . . . . . . . . 389

Daniel H. Gottlieb, The total space of universal fibrations . . . . . . . . . . . .

Yoshimitsu Hasegawa, Integrability theorems for power series expansions of

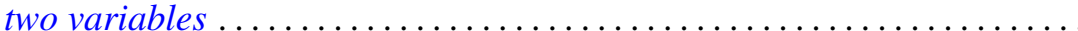

Dean Robert Hickerson, Length of period simple continued fraction expansion of $\sqrt{ } d$

Herbert Meyer Kamowitz, The spectra of endomorphisms of the disc algebra.

Dong S. Kim, Boundedly holomorphic convex domains

Daniel Ralph Lewis, Integral operators on $\mathscr{L}_{p}$-spaces ...

John Eldon Mack, Fields of topological spaces . . . . . . . . .

V. B. Moscatelli, On a problem of completion in bornology

Ellen Elizabeth Reed, Proximity convergence structures. .

Ronald C. Rosier, Dual spaces of certain vector sequence spaces .

Robert A. Rubin, Absolutely torsion-free rings

Leo Sario and Cecilia Wang, Radial quasiharmonic functions . .

James Henry Schmerl, Peano models with many generic classes .

H. J. Schmidt, The $\mathscr{F}$-depth of an $\mathscr{F}$-projector ............

Edward Silverman, Strong quasi-convexity. . . . . . . . . . . . . . . . . 549

Barry Simon, Uniform crossnorms ....................... 555

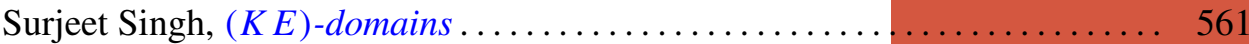

Ted Joe Suffridge, Starlike and convex maps in Banach spaces . . . . . . . . 575

Milton Don Ulmer, $C$-embedded $\Sigma$-spaces . . . . . . . . . . . . . . . . 591

Wolmer Vasconcelos, Conductor, projectivity and injectivity . . . . . . . . . 603 